\title{
The Comparison of Helical-IMRT, Direct-IMRT and 3D Radiotherapy Modalities in Breast Radiotherapy Planning
}

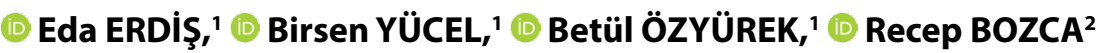 \\ 'Department of Radiation Oncology, Sivas Cumhuriyet University Faculty of Medicine, Sivas-Turkey \\ ${ }^{2}$ Department of Radiation Oncology, Başkent University Faculty of Medicine, Adana-Turkey
}

\begin{abstract}
OBJECTIVE
This study aims to compare dosimetric 3D-CRT, Tomo-helical IMRT and Direct IMRT methods in intact breast irradiation.

METHODS

Radiotherapy was planned with three different techniques simulated images of 30 breast cancer patients with negative lymph nodes who underwent breast-conserving surgery.

\section{RESULTS}

The maximum and minimum doses, homogeneity and conformity index and cold-zone volumes were found to be different in all three techniques $(\mathrm{p}<0.001)$. The PTV boost maximum doses and homogeneity index were found to be different $(\mathrm{p}<0.001)$. The maximum non-PTV dose $(110 \%$ volume) and the hot zone volumes grasped by these doses, the V 5 volume of the body and V5 volumes, outer of PTV were found to be statistically significant for all three techniques $(\mathrm{p}<0.001)$. A statistically significant difference was found between the three techniques concerning the ipsilateral lung V5 and V20 volumes, the contralateral lung V5 and V10 volumes and the counter breast maximum and mean doses $(\mathrm{p}<0.001)$. The mean and V10 volume of the heart was statistically significant $(\mathrm{p}<0.001)$, while the left breast irradiation and mean values were not statistically significant between the three techniques $(\mathrm{p}=0.529)$. However, there was a statistically significant difference in the V10 volume of the left breast irradiation between the three techniques $(\mathrm{p}=0.033)$.
\end{abstract}

\section{CONCLUSION}

According to 3D-CRT, it is possible to achieve better dose distribution and dose homogeneity with Tomo-helical and direct IMRT. Low dose volumes are high with tomo-helical IMRT. Tomo-helical IMRT planning provides better ipsilateral lung and heart doses but causes an increase in heart dose/contralateral breast and for right breast irradiation causes an increase in heart dose.

Keywords: Breast cancer; tomotherapy-direct IMRT; tomotherapy-helical IMRT; three-dimensional conformal radiotherapy.

Copyright $\odot$ 2020, Turkish Society for Radiation Oncology

\section{Introduction}

Breast cancer is the most common cancer in women. [1] Radiotherapy (RT) after breast-conserving surgery in early-stage breast cancer patients has been shown to have a positive effect on local control and survival.[2]

Conventional radiotherapy devices can deliver a wide range of radiation in only a few angles. However,

\author{
Dr. Eda ERDiş \\ Sivas Cumhuriyet Üniversitesi Tıp Fakültesi, \\ Radyasyon Onkolojisi Anabilim Dalı, \\ Sivas-Turkey \\ E-mail: dr.erdiseda@hotmail.com
}


with the advances in technology, various methods, such as three-dimensional conformal RT (3D CRT), density-regulated RT (IMRT) and non-IMRT conformal techniques (forward-IMRT, Direct Aperture Optimization, and Hybrid IMRT), have been applied in breast cancer treatment.

The Tomo-helical/direct IMRT method, which is a form of IMRT application, has been found in the treatment of breast cancer.[3-5] The tomography is a computed tomography-guided IMRT, and the treatment rays are helically optimized from all angles, allowing for a prescribed dose. Instead of a single radiation beam, it treats many small beamlets with rapidly moving micro multileaf collimators. Thus, different doses enable the preservation of healthy tissues. [6] Tomo-helical IMRT 6-MV is a combination of the linear accelerator and computed tomography, which allows IMRT application and megavoltage imaging for daily set-up controls. Tomo-Direct IMRT is the classic IMRT application, but the patient is treated by sliding it with simultaneous table movements along the beam area.

In the study comparing the plan quality and secondary malignancy risks of five different treatment techniques (volumetric modulated arc therapy, IMRT, 3D-CRT, Tomo-helical, and Tomo-direct IMRT), the Tomo-helical IMRT plan provides a better plan quality compared to other techniques and reported that secondary malignancy provides the lowest lifelong attributable risk for normal tissues (internal organs, such as the ipilateral lung and non-field organs). Therefore, it is thought that irradiation with helical tomotherapy may be a better treatment method in young patients with a long life.[7]

The present study aims to compare the dosimetric methods of 3D-CRT, Tomo-helical IMRT and Tomo-
Direct IMRT in intact breast irradiation of patients undergoing BCS for breast cancer.

\section{Materials and Methods}

This study was approved by the institutional ethics committee and was conducted in accordance with the ethical standards laid down in the 1964 Declaration of Helsinki.

Radiotherapy was planned with three different techniques in 30 breast cancer patients with negative lymph nodes who underwent breast-conserving surgery. A total of 90 plans were created: three plans per patient (3D-CRT, Tomo-helical and direct plans).

\section{Treatment Planning}

All patients underwent computed tomography (CT) with a wingboard in the supine position for treatment planning. They had a planning CT scan at a $3-\mathrm{mm}$ slice thickness. The planning target volume was contoured according to the Radiation Therapy Oncology Group (RTOG) atlas.[8]

The breast contour included all glandular breast tissue. The cranial border was contoured below the head of the clavicle at the insertion of the second rib. The lateral border was defined by the midaxillary line. The medial border was defined at the edge of the sternum. The caudal border was defined by the loss of breast tissue, and the anterior border was extracted a few millimeters $(3 \mathrm{~mm})$ from the surface of the skin.

The lumpectomy cavity included seroma, surgical clips, and notable differences in the glandular breast tissue. The lumpectomy cavity with a margin of 0.5$1 \mathrm{~cm}$ was defined PTVboost (planning target volume). Figure 1 shows an exemplary breast and lumpectomy cavity contour.
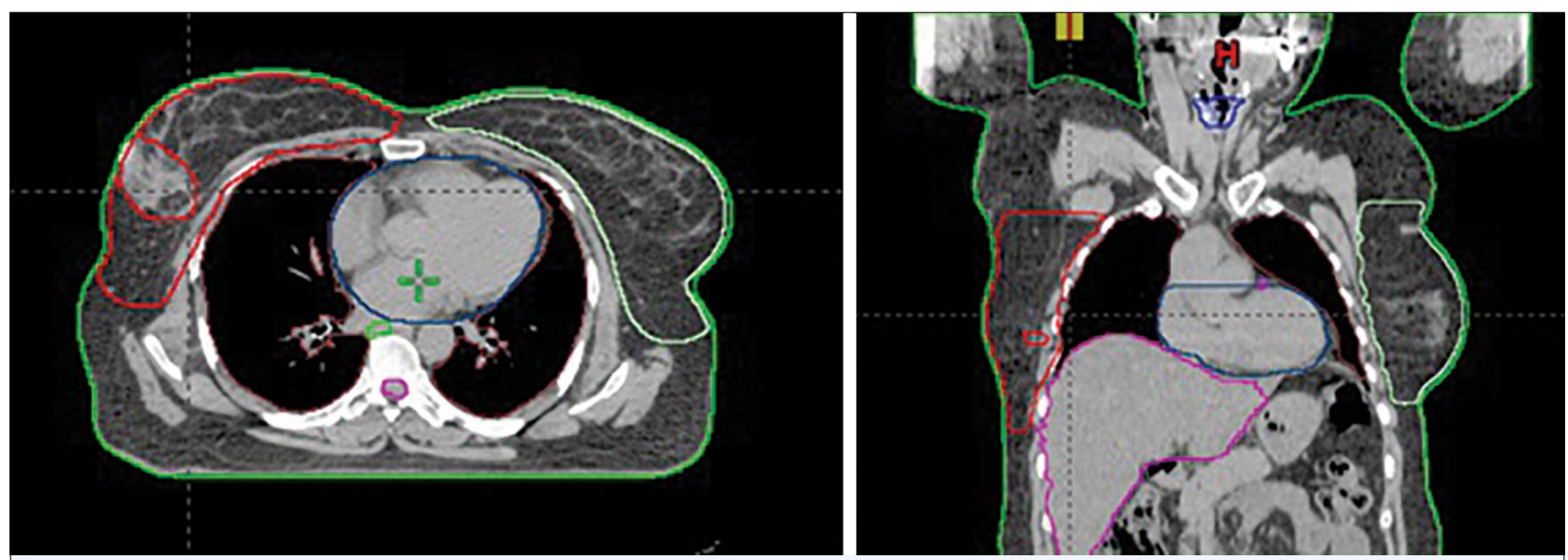

Fig. 1. The breast contouring. (a) axial slide (b) koronal slide. 
The heart, lungs, spinal cord, and contralateral breast were contoured as Organs at Risk (OAR). Figure 1 shows an exemplary breast contour.

Three-dimensional conformal radiotherapy planning was performed using the Eclipse (ver. 8.6; Varian Medical Systems, Inc. Palo Alto, CA, USA) software program. Intact breast and boost fields were carried out using a 6-18 MV photon beam. The 3D-CRT plan consisted of opposed tangential beams and added subfields created by MLC for dose compensation (FiF). In the FiF segmental IMRT technique, the same beam orientation as 3D-CRT can be used. In the FiF technique, the homogeneous dose distribution was obtained by MLC instead of wedge filters by closing the hot areas and adding new sub-areas to the cold regions. In the planning of the boost zone, the electron field or minitangential field technique with appropriate energy was used.

The tomotherapy plans were performed using a Tomo-H VoLO planning system (Accuray Inc. Madison, WI, USA). All Tomo-helical and direct IMRT plans were generated using the same constraints (field width: $2.5 \mathrm{~cm}$, pitch: 0.215 , modulation factor: $2-5$ ). The Tomo-Direct IMRT technique uses a static gantry position. The dynamic collimator is similar to the classic IMRT using a sweeping window, and it is shifted through simultaneous table movements along the patient beam area. In the direct IMRT plans, a 4-6 area and the $2.5 \mathrm{~cm}$-wide plan was made. In the Tomo-helical IMRT technique, the target volume irradiation was carried out helically with a fan-shaped narrowed fan beam in continuous rotation. In the helical IMRT plans, a critical block was applied to the critical organs, and an area of $2.5 \mathrm{~cm}$ was planned.

The target volume coverage was selected as D98 in both plans. In these treatment plans, $98 \%$ of the dose defined for the PTV was provided. For all three techniques, 50 Gy was planned in 25 fractions in the intact breast and $60 \mathrm{~Gy}$ in 30 fractions in the tumor bed. The dose limitation of the critical organs was ipsilateral lung V5 $<60 \%$ and V $20<22 \%$, heart mean $<5$ Gy, and contralateral breast mean $<3 \mathrm{~Gy}$.

\section{Evaluation of Radiotherapy Plans}

All volumes were compared with the dose-volume histograms. For the target volumes, the Dmax, Dmin, cold zone (cc), conformity index (CI), and homogeneity index (HI) values were compared. The dose suitability and homogeneity were measured and estimated according to the International Radiation Unit and Measurement Commission (ICRU) 83.[9]
In radiotherapy, a homogeneous dose distribution is aimed at the target, and the evaluation is made by looking at the doses taken by $2 \%$ (D2), $50 \%$ (D50), and 98\% (D98) of the PTV, whether the defined dose encapsulates the target (HI=D2-D98/D50) or not. As this value approaches zero, the homogeneity of the dose distribution in the PTV appears to be ideal.

According to ICRU 83, the conformity index is calculated by dividing the volume of PTV wrapped by the specified reference dose by the total PTV volume, and the reference dose is expected to envelop the entire PTV. The ideal value of CI is 1 . A CI close to 1 indicates that the dose covered by the target volume is good. The PTV volume, which receives $95 \%$ of the defined dose, is obtained by the PTV division ( $\mathrm{CI}=\mathrm{TV}$ prescribed/ PTV).

For the critical organs, the mean dose and V10 volume of the heart, the mean dose of the ipsilateral and contralateral lung, the V5, V10, and V20 volumes, and the maximum and mean dose of the contralateral breast were used to compare the techniques.

\section{Statistical Analysis}

We performed all analyses using the SPSS (Statistical Package for Social Sciences) software (Version 22.0; SPSS Inc., Chicago, IL, USA). Frequency analyses and Kruskal-Wallis tests were used to statistically analyze the data. We considered the differences to be statistically significant at $\mathrm{p}$-value $<0.05$.

\section{Results}

\section{Target and non-PTV Volumes}

There was a statistically significant difference between the three techniques concerning the maximum and minimum doses, the homogeneity and conformity index, and the cold-zone volumes within the PTVbreast $(\mathrm{p}<0.001)$. When the conformity index was evaluated, it was observed that the helical and direct plans were similar and statistically lower than the 3D-CRT $(p=0.002)$. However, HI was the lowest value in the Tomo-helical IMRT $(\mathrm{p}<0.001)$. Tomo-IMRT was superior to conformal techniques.

A statistically significant difference was found between the three techniques concerning the PTVboost maximum doses and homogeneity index $(\mathrm{p}<0.001)$. Dose homogeneity was best achieved using the Tomohelical IMRT technique. The cold zone was not present in all three techniques.

The maximum non-PTV dose ( $110 \%$ area volume) and the hot-zone volumes grasped by these doses were 
statistically significant $(\mathrm{p}<0.001)$. The V5 volume of the body and the V 5 area, except the PTV, were found to be statistically significant for all three techniques $(p<0.001)$. 3D-CRT was superior for the V5 volume of the body.

Comparisons of Tomo-Direct IMRT, Tomo-helical IMRT, and 3D-CRT planning (target volumes, out of PTV volumes and body) are shown in Table 1.

\section{Organ at Risk}

The V5 and V20 Gy dose volumes of the ipsilateral lung doses were different and statistically significant in all three techniques $(p<0.001)$. In the Direct-IMRT planning, the V5 value was lower than the other two techniques $(\mathrm{p}<0.001)$.

The V5 and V10 Gy volumes of the contralateral lungs were found to be different between the techniques, and the V20 Gy volume was not determined in the plans made with all three techniques $(\mathrm{p}<0.001)$.

In the mean-breast irradiation, the mean and 10 Gy field volume of the heart was statistically significant $(p<0.001)$, while the left-breast irradiation, mean values were not statistically significant between the three techniques $(p=0.529)$. However, there was a statistically significant difference in the V10 and V25 Gy dose volumes of the left-breast irradiation between the three techniques $(\mathrm{p}=0.033$ and $\mathrm{p}=0.014)$.
There was a statistically significant difference between the three techniques for the maximum and mean dose of the contralateral breast $(\mathrm{p}<0.001)$.

Table 2 shows the radiation doses received by the organs at risk comparisons of the Tomo-Direct IMRT, Tomo-helical IMRT, and 3D-CRT plannings.

\section{Discussion}

With rapidly developing technology, the techniques used in RT also change over time. As the treatment devices changed, the treatment techniques and dose calculation algorithms given to the patient also changed. Although each treatment technique has its own advantages and disadvantages, the techniques that enable us to protect the critical organs better and to give higher doses are preferred because they are more successful in controlling the tumor area. With the use of computed tomography in radiotherapy planning, more homogeneous and conformal dose distributions have begun to be obtained. In 3D treatment planning, the tumor volume received a homogeneous dose, while the risky organs were preserved. After the 3D treatment planning, intensity-adjusted software was used. In the 2000s, clinical studies comparing different techniques were conducted on the use of sophisticated new

\begin{tabular}{|c|c|c|c|c|}
\hline & $\begin{array}{c}\text { Tomo-Direct IMRT } \\
n=30\end{array}$ & $\begin{array}{l}\text { Tomo-Helical IMRT } \\
\qquad n=30\end{array}$ & $\begin{array}{c}\text { 3D-CRT } \\
n=30\end{array}$ & $\mathbf{p}$ \\
\hline \multicolumn{5}{|l|}{ PTV-tangential dose } \\
\hline Maximum (Gy) & 65.0 & 64.3 & 67.3 & $<0.001$ \\
\hline Minimum (Gy) & 45.4 & 45.8 & 37.2 & $<0.001$ \\
\hline Cold region (cc) & 0.2 & 0.24 & 3.5 & $<0.001$ \\
\hline Conformity index & 0.98 & 0.98 & 0.96 & 0.002 \\
\hline Homogeneity index & 0.21 & 0.16 & 0.28 & $<0.001$ \\
\hline \multicolumn{5}{|l|}{ PTV-boost dose } \\
\hline Maximum (Gy) & 65.1 & 64.3 & 66.7 & $<0.001$ \\
\hline Minimum (Gy) & 58.0 & 58.3 & 56.8 & 0.183 \\
\hline Cold region (cc) & 0 & 0 & 0 & - \\
\hline Conformity index & 0.98 & 0.98 & 0.99 & 0.070 \\
\hline Homogeneity index & 0.07 & 0.05 & 0.08 & $<0.001$ \\
\hline \multicolumn{5}{|l|}{ Out of PTV } \\
\hline Maximum (Gy) & 69.0 & 64.5 & 67.3 & $<0.001$ \\
\hline Hot region (cc, $110 \%$ alan volume) & 60.2 & 0.0 & 46 & $<0.001$ \\
\hline V5 (cc) & 2285 & 5948 & 2090 & $<0.001$ \\
\hline \multicolumn{5}{|l|}{ Body } \\
\hline V5 (\%) & 9.15 & 17.5 & 9.3 & $<0.001$ \\
\hline
\end{tabular}

*Median values for all variables were noted; 3D-CRT: 3-dimensional radiotherapy, IMRT: Intensity-modulated radiotherapy 
Table 2 Comparisons of the tomo-direct IMRT, tomo-helical IMRT and 3D-CRT planning (OAR)

\section{Tomo-direct IMRT \\ $\mathrm{n}=\mathbf{3 0}$}

Ipsilateral lung dose

V5 (\%)

V10 (\%)

V20 (\%)

Contralateral lung dose

V5 (\%)

V10 (\%)

Cardiac dose (right-sided breast)

Mean (Gy)

V10 (\%)

Cardiac dose (left-sided breast)

Mean (Gy)

V10 (\%)

V25 (\%)

Contralateral breast dose

Maximum (Gy)

Mean (Gy)

\section{Tomo-helical IMRT}

$n=30$

30.5

21.0

11.3

23.8

3.2

4.3

2.6

5.4

7.3

0

7.7

2.2

\section{D-CRT}

$n=30$

p

$\begin{array}{cccc}4.8 & 5.4 & 5.6 & 0.529 \\ 10.6 & 7.3 & 10.0 & 0.033 \\ 4.95 & 0 & 7.15 & 0.014 \\ & & & \\ 3.5 & 7.7 & 7.9 & <0.001 \\ 0.3 & 2.2 & 0.7 & <0.001\end{array}$

*Median values for all variables were noted; 3D-CRT: 3-dimensional radiotherapy, IMRT: Intensity-modulated radiotherapy; OAR: Organs at risk

techniques, such as Tomo-helical IMRT. Many clinical studies have compared dosimetric 3D-CRT and IMRT techniques. Various publications have reported that IMRT provides adequate and more homogeneous dose distribution to the target compared to 3D-CRT, and it provides better protection of the heart, lung, and counter-breast.[10-15] Limiting doses of the lungs, hearts, and breasts adjacent to the breast is very important in reducing side effects. Accurate implementation of the treatment plan is essential to treatment success. It is also important to verify that the planned dose at the target is administered as planned.

\section{Target and out of PTV Volumes}

In this study, the 3D-CRT, Tomo-helical and TomoDirect IMRT techniques were compared in 30 lymphnode-negative patients undergoing lumpectomy. When the results of these three techniques were analyzed, it was observed that the tomotherapy techniques improved the target volume better than 3D-CRT, but the dose homogeneity was best achieved with the Tomo-helical IMRT technique. Again, the maximum and minimum doses and the cold-zone values in the target volume were obtained by the best tomotherapy techniques (Fig. 2). However, the low-dose volume in the body was highest in the Tomo-helical IMRT (almost twice as high as the other two methods). Figure 3 shows the low-dose (V5) area volumes of the three techniques.
When the figure is examined, it is seen that the V5 dose volumes are larger in Tomo-helical IMRT planning.

Goddu et al. compared helical tomotherapy with 3D-CRT planning. The chest wall/breast, supraclavicular and intramammary lymph nodes were contoured and planned for both techniques in 10 lymph-nodepositive left-breast cancer patients. It has been shown that Tomo-helical IMRT envelops the target better than 3D-CRT and provides better dose homogeneity.[16]

Reynders et al. compared 3D-CRT and tomotherapy (helical and direct) RT planning in eight breast cancer patients. In the study, 6-7 plans were prepared for each patient in the supine and prone position of the patients, left-right tumor, lymph-node positive, lymphnode negative, MRM and MKC were performed and three techniques were compared. Researchers have reported that Tomo-helical and direct planning provides a dose distribution equal to or better than 3D-CRT in the target volume and organs to be protected.[17]

In the dosimetric study conducted by Schubert et al., the left whole-breast RT planning was performed with different IMRT techniques. They compared 3DCRT planning. In this study, the left-breast RT planning of 10 patients was performed with the 3D-CRT, Tomo-helical, Tomo-direct IMRT, inverse-IMRT, and forward-IMRT techniques. As a result, the maximum doses of the target volume were found to be lower using the IMRT techniques than the 3D-CRT. All the 

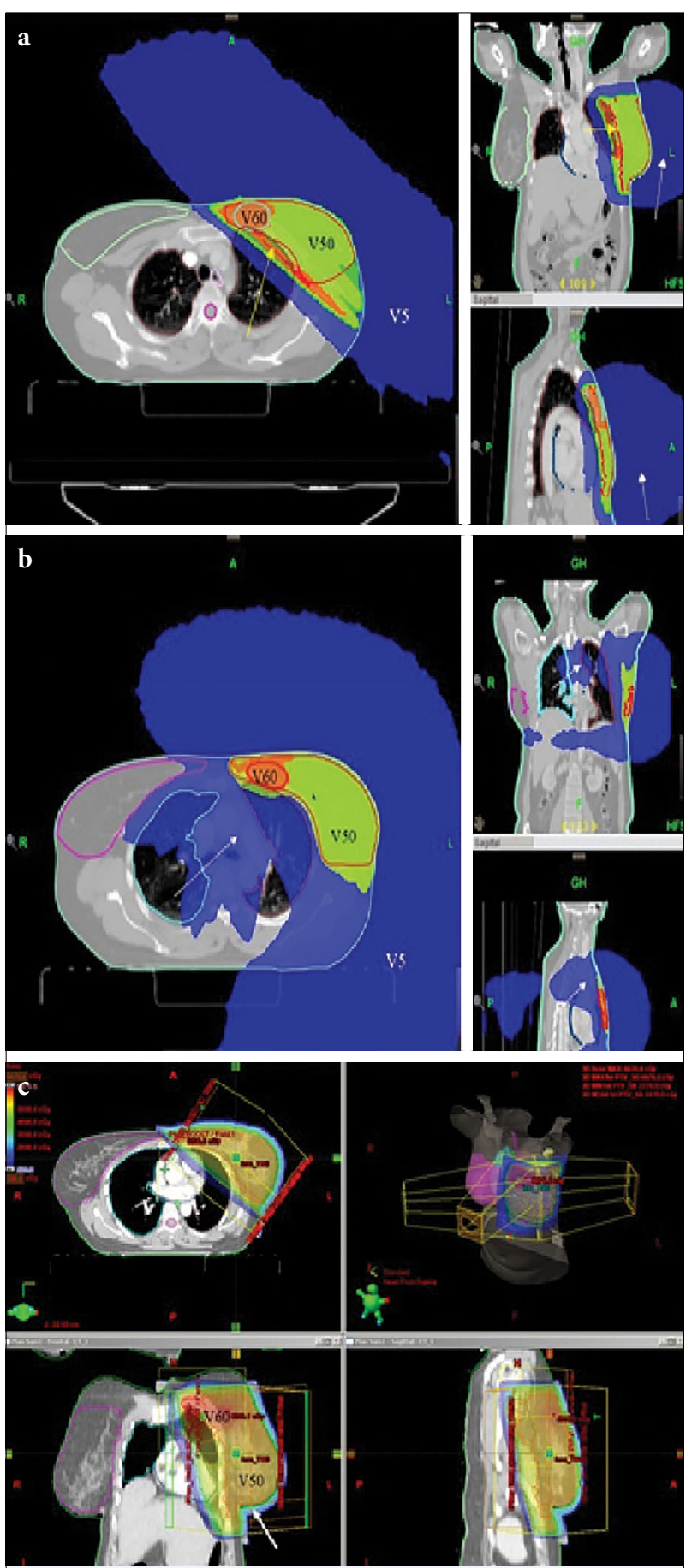

Fig. 2. The dose distribution of three different techniques (V5, V50, V60 volumes). (a) The dose distribution of direct-TomoTherapy. The white arrows point to low (5 Gy) and the yellow arrow point to high dose (\%110) volumes. (b) The dose distribution of helical-TomoTherapy. The white arrows point to low dose (5Gy) volume. (c) The dose distribution of 3D-CRT. The white arrows point to low dose (5Gy) volume. methods evaluated provided adequate coverage of the dose in the target breast. However, the dose homogeneity was found to be more successful using the Tomohelical and Tomo-direct IMRT techniques than 3DCRT.[18]

Shiau et al. compared Tomo-helical IMRT and hybrid IMRT in 30 early-stage left-breast cancer patients. The target coverage of both methods was similar. However, CI and HI were better detected in Tomo-helical IMRT.[19]

In a letter written by Arsene-Henry et al., it states that it covers the target with adequate and homogeneous dose distribution and reduces OAR doses. However, they stated that the risk of a secondary malignancy might be increased because of the low dose volumes taken from the counter lung, the opposite breast, and other normal tissues in Tomo-helical IMRT.[20]

Ashenafi et al. compared chest-wall irradiation after a mastectomy with Tomo-helical IMRT and a conventional method (electron-photon beam mixture). In their study, it was shown that the dose homogeneity of the target volume was better achieved in Tomo-helical IMRT, but the V5 and V25 volumes of the body were significantly wider in the planning of Tomo-helical IMRT $(\mathrm{p}<0.001)$. In addition, they found that a secondary cancer complication probability was significantly higher for Tomo-helical IMRT planning. [21] Similarly, in our study, the volumes of V5 doses received by the body were found to be higher than the other two methods. In the three methods we compared, the lowest V5 volume was obtained in 3D-CRT (Fig. 3).

\section{Organs at Risk}

In our study, the dose of the ipsilateral lung V5 was found to be statistically significantly lower than Tomohelical and Direct IMRT compared to 3D-CRT. The V20 dose of the ipsilateral lung was the lowest Tomo-helical IMRT compared to the other two methods. However, the counter-lung V5 and V10 values were determined only in Tomo-helical IMRT, and it was observed that these methods did not take the dose in the other methods. In our study, when the cardiac techniques were compared concerning cardiac doses, the cardiac doses (mean and V10) were higher in the Tomo-helical IMRT planning, which caused wide low-dose volumes in the right-breast planning. However, in the left-breast planning, the V10 and V25 doses receiving volume of the heart was found to be the lowest Tomo-helical IMRT planning. In our study, when the techniques were examined concerning counter-breast doses, the maximum dose of the counter breast was observed in the lowest To- 

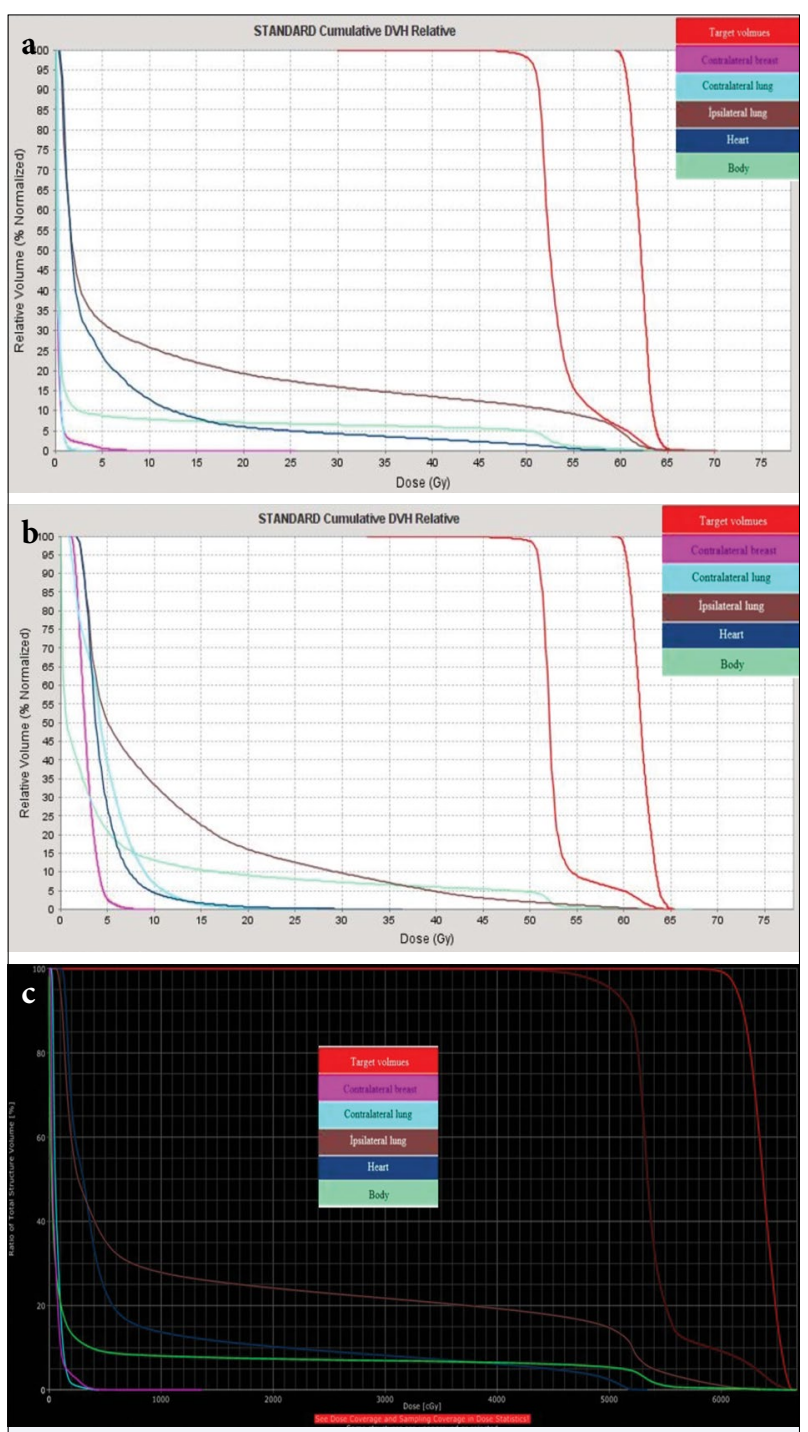

Fig. 3. The dose volume histogram examples of three techniques. (a) The dose volume histogram of Tomo-direct IMRT. (b) The Dose volume histogram of Tomo-helical IMRT. (c) The dose volume histogram of 3D-CRT.

mo-direct IMRT planning. In Tomo-helical IMRT and 3D-CRT planning, the maximum dose of the opposite breast was found to be similar. The mean-breast counter dose was found in the lowest Tomo-direct IMRT, while the highest counter breast mean dose was found in Tomo-helical planning. Figure 3 shows the DVH examples of the three techniques.

In the dosimetric study of Schubert et al., comparing the dose distribution and risky organ protection provided by modern RT techniques to the target, all the techniques (3D-CRT, Tomo-helical, Tomo-direct IMRT, inverse-IMRT, forward-IMRT, hybrid IMRT) breast) provided adequate doses. The maximum doses of the ipsilateral lung and heart were found to be the lowest in Tomo-helical IMRT, but the mean doses of both structures were found to be highest in Tomo-helical IMRT. Compared to Inverse-IMRT, Tomo-direct IMRT, and 3DCRT, the IMRT techniques reduced the mean and maximum doses of the ipsilateral lung. Tomo-helical, tomo-direct and inverse-IMRT were able to reduce the maximum doses in the target and normal tissues, but low-dose volumes of normal tissue were found to be high in Tomo-helical IMRT.[18]

In another dosimetric study comparing Tomo-helical IMRT and Tomo-3D-CRT, which are the options of tomotherapy, it was observed that Tomo-helical IMRT was superior concerning contralateral breast and heart doses.[22]

In a study in which Goddu et al. compared Tomohelical IMRT and 3D-CRT planning and aimed to protect lung, heart and breast against, according to 3DCRT planning, left lung V20, mean dose of heart and right breast dose It was found to be lower.[16]

Coon et al. compared 3D-CRT, IMRT, and Tomohelical IMRT in 15 left-breast cancer patients with unfavorable heart anatomy after a lumpectomy. The mean heart dose and ventricular volume dose were lower in IMRT and Tomo-helical IMRT planning than 3DCRT. However, the counter-breast dose was found to be higher in Tomo-helical IMRT.[23]

In a 2015 dosimetric study, five different methods, volumetric modulated arc therapy, forward-IMRT, inverse-IMRT, 3D-CRT, and Tomo-helical IMRT, were compared. In this study, 15 patients with left-breast carcinoma and who underwent MKC were evaluated. Helical IMRT was found to have a lower organ dose risk, including heart, ipsilateral lung, and LAD. However, the volumes receiving low-dose Tomo-helical IMRT are higher than other methods. [24]

In their dosimetric study (3D-CRT vs. Tomo-helical and direct IMRT), Reynders et al. reported that tomotherapy techniques could achieve an equal or better dose distribution to 3D-CRT in OAR.[17]

\section{Conclusion}

According to 3D-CRT, it is possible to achieve better dose distribution and dose homogeneity with tomotherapy techniques (Tomo-helical and direct IMRT), but keep in mind that the body's low dose volumes are high, especially with Tomo-helical IMRT.

For ipsilateral lung and heart doses, Tomo-helical IMRT planning provides good protection but causes an 
increase in heart dose in the contralateral breast, contralateral lung and right breast irradiation (due to the high body's low-dose volumes). This disadvantageous situation in OAR with Tomo-helical IMRT seems to be solved by Tomo-direct IMRT planning.

Peer-review: Externally peer-reviewed.

Conflict of Interest: The authors have no conflicts of interest to declare.

Ethics Committee Approval: The study protocol was approved by the institutional review board. (Number: 201704/14, Date: 19.04.2017)

Financial Support: The authors declared that this study has received no financial support.

Authorship contributions: Concept - E.E.; Design - E.E.; Supervision - E.E.; Funding - None; Materials - B.Ö.; Data collection and/or processing - R.B.; Data analysis and/or interpretation - E.E.; Literature search - E.E.; Writing - E.E.; Critical review - B.Y.

\section{References}

1. Jemal A, Bray F, Center MM, Ferlay J, Ward E, Forman D. Global cancer statistics. CA Cancer J Clin 2011;61(2):69-90.

2. Early Breast Cancer Trialists' Collaborative Group (EBCTCG), Darby S, McGale P, Correa C, Taylor C, Arriagada R, et al. Effect of radiotherapy after breastconserving surgery on 10-year recurrence and 15-year breast cancer death: meta-analysis of individual patient data for 10,801 women in 17 randomised trials. Lancet 2011;378(9804):1707-16.

3. Donovan EM, James H, Bonora M, Yarnold JR, Evans PM. Second cancer incidence risk estimates using BEIR VII models for standard and complex external beam radiotherapy for early breast cancer. Med Phys 2012;39(10):5814-24.

4. Lee B, Lee S, Sung J, Yoon M. Radiotherapy-induced secondary cancer risk for breast cancer: 3D conformal therapy versus IMRT versus VMAT. J Radiol Prot 2014;34(2):325-31.

5. Abo-Madyan Y, Aziz MH, Aly MM, Schneider F, Sperk E, Clausen S, et al. Second cancer risk after 3D-CRT, IMRT and VMAT for breast cancer. Radiother Oncol 2014;110(3):471-6.

6. O’Donnell H, Cooke K, Walsh N, Plowman PN. Early experience of tomotherapy-based intensity-modulated radiotherapy for breast cancer treatment. Clin Oncol (R Coll Radiol) 2009;21(4):294-301.

7. Han EY, Paudel N, Sung J, Yoon M, Chung WK, Kim DW. Estimation of the risk of secondary malignancy arising from whole-breast irradiation: comparison of five radiotherapy modalities, including TomoHDA. Oncotarget 2016;7(16):22960-9.

8. Radiation Therapy Oncology Group (RTOG) Breast Cancer Contouring Atlas, Available at: http:// www.rtog.org./Corelab/ContouringAtlases/BreastCancerAtlas.aspx.

9. International Commission on Radiation Units and Measurements. Prescribing, Recording, and Reporting Photon-Beam Intensity-Modulated Radiation Therapy (IMRT): ICRU Report 83, 2010. Available at: https://academic.oup.com/jicru/article-abstract/10/1/ NP/910527?redirectedFrom=fulltext. Accessed Mar 17, 2020.

10. Vicini FA, Sharpe M, Kestin L, Martinez A, Mitchell CK, Wallace MF, et al. Optimizing breast cancer treatment efficacy with intensity-modulated radiotherapy. Int J Radiat Oncol Biol Phys 2002;54(5):1336-44.

11. Lo YC, Yasuda G, Fitzgerald TJ, Urie MM. Intensity modulation for breast treatment using static multi-leaf collimators. Int J Radiat Oncol Biol Phys 2000;46(1):187-94.

12. Krueger EA, Fraass BA, Pierce LJ. Clinical aspects of intensity-modulated radiotherapy in the treatment of breast cancer. Semin Radiat Oncol 2002;12(3):250-9.

13. Hurkmans CW, Cho BC, Damen E, Zijp L, Mijnheer BJ. Reduction of cardiac and lung complication probabilities after breast irradiation using conformal radiotherapy with or without intensity modulation. Radiother Oncol 2002;62(2):163-71.

14. Andratschke N, Maurer J, Molls M, Trott KR. Late radiation-induced heart disease after radiotherapy. Clinical importance, radiobiological mechanisms and strategies of prevention. Radiother Oncol 2011;100(2):160-6.

15. Offersen B, Højris I, Overgaard M. Radiation-induced heart morbidity after adjuvant radiotherapy of early breast cancer - Is it still an issue?. Radiother Oncol 2011;100(2):157-9.

16. Goddu SM, Chaudhari S, Mamalui-Hunter M, Pechenaya OL, Pratt D, Mutic S, et al. Helical tomotherapy planning for left-sided breast cancer patients with positive lymph nodes: comparison to conventional multiport breast technique. Int J Radiat Oncol Biol Phys 2009;73(4):1243-51.

17. Reynders T, Tournel K, De Coninck P, Heymann S, Vinh-Hung V, Van Parijs H, et al. Dosimetric assessment of static and helical TomoTherapy in the clinical implementation of breast cancer treatments. Radiother Oncol 2009;93(1):71-9.

18. Schubert LK, Gondi V, Sengbusch E, Westerly DC, Soisson ET, Paliwal BR, et al. Dosimetric comparison of left-sided whole breast irradiation with 3DCRT, forward-planned IMRT, inverse-planned IMRT, he- 
lical tomotherapy, and topotherapy. Radiother Oncol 2011;100(2):241-6.

19. Shiau AC, Hsieh CH, Tien HJ, Yeh HP, Lin CT, Shueng PW, et al. Left-sided whole breast irradiation with hybrid-IMRT and helical tomotherapy dosimetric comparison. Biomed Res Int 2014;2014:741326.

20. Arsene-Henry A, Fourquet A, Kirova YM. Evolution of radiation techniques in the treatment of breast cancer (BC) patients: From 3D conformal radiotherapy (3D CRT) to intensity-modulated RT (IMRT) using Helical Tomotherapy (HT). Radiother Oncol 2017;124(2):333-4.

21. Ashenafi M, Boyd RA, Lee TK, Lo KK, Gibbons JP, Rosen II, Fontenot JD, Hogstrom KR. Feasibility of postmastectomy treatment with helical TomoTherapy. Int J Radiat Oncol Biol Phys 2010;77(3):836-42.
22. Yadav P, Yan Y, Ignatowski T, Olson A. Dosimetric aspects of breast radiotherapy with three-dimensional and intensity-modulated radiotherapy helical tomotherapy planning modules. Med Dosim 2017;42(1):42-6.

23. Coon AB, Dickler A, Kirk MC, Liao Y, Shah AP, Strauss JB, et al. Tomotherapy and multifield intensity-modulated radiotherapy planning reduce cardiac doses in left-sided breast cancer patients with unfavorable cardiac anatomy. Int J Radiat Oncol Biol Phys 2010;78(1):104-10.

24. Haciislamoglu E, Colak F, Canyilmaz E, Dirican B, Gurdalli S, Yilmaz AH, et al. Dosimetric comparison of left-sided whole-breast irradiation with 3DCRT, forward-planned IMRT, inverse-planned IMRT, helical tomotherapy, and volumetric arc therapy. Phys Med 2015;31(4):360-7. 